\title{
ERAS Optimization of Cluster Intervention Strategies in Perioperative Period of Acute Stanford Type A Aortic Dissection
}

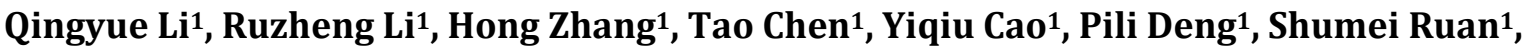 \\ Chunqiong Lin', Nanyao Chen ${ }^{2}$
}

${ }^{1}$ Hainan General Hospital, Haikou, China

${ }^{2}$ The Fourth People's Hospital of Haikou, Haikou, China

Email: g2002m@163.com

How to cite this paper: Li, Q.Y., Li, R.Z., Zhang, H., Chen, T., Cao, Y.Q., Deng, P.L., Ruan, S.M., Lin, C.Q. and Chen, N.Y. (2020) ERAS Optimization of Cluster Intervention Strategies in Perioperative Period of Acute Stanford Type A Aortic Dissection. Health, 12, 1457-1467. https://doi.org/10.4236/health.2020.1211106

Received: October 25, 2020

Accepted: November 24, 2020

Published: November 27, 2020

Copyright $\odot 2020$ by author(s) and Scientific Research Publishing Inc. This work is licensed under the Creative Commons Attribution International License (CC BY 4.0).

http://creativecommons.org/licenses/by/4.0/ (c) (i) Open Access

\begin{abstract}
Accelerated rehabilitation surgery (ERAS) can promote postoperative recovery and reduce postoperative complications by minimizing surgically related stress, and its efficacy and safety in acute aortic dissection surgery have been demonstrated. However, due to the particularity of acute aortic dissection, studies on the application of accelerated rehabilitation surgery in acute aortic dissection are relatively rare and there are great differences among different studies. This study adopts the method of evidence-based nursing practice accelerate rehabilitation cluster of surgical nursing strategy developed and applied to the patients with primary acute aortic dissection and adopts "accelerate rehabilitation cluster of surgical nursing strategy" and "accelerated rehabilitation cluster of surgical nursing strategies in patients with acute aortic dissection in clinical intervention", the new nursing concept and organic combination of traditional nursing measures, using the optimal cluster nursing intervention mode, to explore the safety, effectiveness and feasibility of the intervention mode in the perioperative period of the original acute aortic dissection in patients' cluster intervention, to accelerate the rehabilitation surgery in acute aortic dissection in the clinical care to provide a scientific basis for the operation.
\end{abstract}

\section{Keywords}

Accelerated Rehabilitation Surgery, Acute Stanford, Type A Aortic

Dissection, Cluster Care

\section{Introduction}

Aortic dissection (AD) is an acute aortic disease with acute onset, critical condi- 
tion, rapid progression and high mortality. Accelerate rehabilitation surgery (ERAS) means to make patients' rapid recovery, the perioperative adopts a series of effective optimization measures confirmed by evidence-based medical evidence, to relieve the patients' psychological and physical trauma stress reaction, achieve rapid rehabilitation, reduce complications, shorten hospitalization time, reduce the risk of readmission and mortality risk, etc. [1]. Acute aortic syndrome (Acuteaorticsyndrome, AAS) is a group of heterogeneous disease, clinical symptoms, similar performance as the chief complaint, are chest pain more, also called acute chest pain syndrome, including the aortic dissection (Aorticdissection, AD), the aortic intramural hematomas (IMH) Intramuralaortichematoma, and aortic penetrating ulcer (Penetrating Atherosclerotic Aorticulcer, PAU), the rapid onset of the disease. The incidence of complications and mortality is very high, if not promptly treated, often will die of aortic rupture, hemorrhagic shock, sudden death, etc. Therefore, increasing the understanding of the pathogenesis of AAS plays an important role in the early diagnosis, reasonable treatment and prevention of complications of AAS. Aortic dissection is referring to the aorta under the effect of a series of external factors, such as high blood pressure, trauma, etc. Based on the presence or absence of lesions in the aortic wall itself, there is a rupture in the aortic intima, and the aortic intima rupture of the blood autonomous artery invades the middle layer of the aortic wall. The middle layer of the aorta is continuously torn and longitudially separated, resulting in a coexistence of the true and false lumens of the active vascular lumen. The refined classification of Stanford Type A aortic dissection can be divided into $\mathrm{C}$ type and $\mathrm{S}$ type according to the lesions of the aortic arch. $\mathrm{C}$ type is a complex type, including the following types: 1) The primary intimal rupture is at the arch or its distal end, and the dissection is retrograde to the proximal end of the aortic arch or the ascending aorta; 2) aneurysm formation in the arch or its distal end (diameter: $>5.0 \mathrm{~cm}$ ). There was dissection of brachiocephalic artery. The disease is due to Marfan syndrome. Type S, or simple type, refers to a Stanford Type A aortic dissection that does not have any of the lesions seen in Type $\mathrm{C}$.

[2] for patients with complex Stanford Type A aortic dissection, in order to reduce the occurrence of postoperative aortic dissection and increase the closure rate of postoperative false cavity, total aortic arch replacement + stent elephant nose surgery are generally adopted, while partial arch replacement is generally recommended for simple type. Studies have shown that Sun-style [3] operation method is simple, safe, and the interlayer crevasse is closed to perfect and simplify the operation procedures, also applies to the aorta distal aorta go circuitous situation, operation mortality is low, less postoperative complications, follow-up false cavity closure rate is high, low and reoperation rate, can become the treatment modalities of Stanford type A aortic dissection surgery way new specification.

It has been reported [4] that even after surgery, the mortality rate is as high as $7 \%-13 \%$. In addition, there are many and serious postoperative complications, long hospital stay for patients, high cost, great trauma in cardiopulmonary by- 
pass surgery, perioperative experience of fasting and drinking, pain, various invasive pipes, postoperative complications and other factors, resulting in slow postoperative recovery process, which has a significant impact on patients' physical and mental trauma [5]. The research progress of the optimization of RAS cluster intervention strategy in the perioperative period of acute Stanford type A aortic dissection is reported as follows.

\section{Clinical Application Status of ERAS}

Enhanced Recovery after surgery (ERAS) is a series of perioperative optimization measures confirmed by evidence-based medicine to minimize the stress response of patients and prevent postoperative dysfunction, promote postoperative recovery and reduce postoperative complications, and thus improve patient outcomes [6]. Accelerated rehabilitation surgery was first proposed by Danish abdominal surgeon Kehlet. It was first applied in cardiac surgery, and the most successful clinical application was elective colorectal surgery. Now, it has become the standard treatment for colorectal surgery. Studies have shown that ERAS can reduce the incidence of perioperative complications, shorten postoperative hospitalization time and reduce hospitalization costs [7] [8]. After nearly 20 years of development, ERAS has been extended to many surgical fields such as general surgery, orthopedics, urology, gynecology, cardiothoracic surgery, vascular surgery and plastic surgery [9] [10]. However, the application of ERAS in high-risk surgeries (such as esophagectomy and hepatectomy) still faces challenges, and further studies are needed. ERAS concept runs through all aspects of the perioperative period and is an optimal combination of existing perioperative management measures, which requires the multidisciplinary team cooperation of surgeons, anesthesiologists, nurses and rehabilitation therapists as well as the cooperation of patients and their families.

\subsection{Research Status of ERAS in Gastrointestinal Surgery}

ERAS was first successfully applied in colorectal surgery, and its research level in gastrectomy lags behind that in colorectal surgery. For patients undergoing elective colorectal resection, the incidence of complications was $20 \%$ - 30\% through traditional perioperative nursing, and the postoperative hospitalization duration was 6 - 12 days [11]. The main causes of long hospital stay after colorectal surgery are pain, paralytic ileus, postoperative complications and other organ dysfunction. In order to improve these problems, ERAS concept was developed and the clinical Practice guide of ERAS for rectal surgery was published in 2013 [9] [12]. In China, ERAS concept was first introduced by Academician Li Jieshou in 2007 and applied to gastrectomy [13]. ERAS program focuses on 20 measures [8], including pre-hospital consultation and education, standardized anesthesia, prevention of postoperative ileus, laparoscopic surgery, postoperative nutritional support and early postoperative activities. A number of studies have shown [14] [15] [16] that ERAS can significantly shorten the length of hospital stay and the 
time of first exhaust defecation, reduce the occurrence of postoperative complications, and thus reduce the medical costs without increasing the readmission rate and mortality, indicating that ERAS is safe and effective, and its application in perioperative period of patients undergoing elective colorectal surgery is reasonable. Meanwhile, studies also showed that [17]: ERAS in colorectal surgery could reduce the risk of death by $47 \%$, reduce complications by $47 \%$, and reduce readmission by $20 \%$. ERAS guidelines for gastrectomy were developed by the European Association for Accelerated Rehabilitation Surgery in 2014 [18]. A number of studies have shown [19] [20] [21] [22] that ERAS can promote the recovery of gastrointestinal function in patients with most gastrectomy, shorten the hospitalization time, and do not increase the occurrence of postoperative complications compared with conventional nursing, and ERAS is safe and effective in the application of subtotal gastrectomy. Although there are clear guidelines and solid evidence supporting the application of ERAS in gastrointestinal surgery, the full implementation of ERAS is still confronted with certain difficulties. ERAS schemes in different studies differ greatly, and a relatively complete and unified scheme has not been formed.

\subsection{Research Status of ERAS after Acute Stanford Type A Aortic Dissection}

ERAS protocols after acute Stanford type A aortic dissection included: preoperative health education; Preoperative optimization; Preoperative preparation and preoperative fasting; Perioperative nutrition management; Prevention of thromboembolism; Standardized anesthesia protocols; Improvement of surgical methods; Postoperative analgesia; Early activities: early oral feeding; Prevent postoperative nausea and vomiting. ERAS concept embodies the tenet of the new "patient-centered" medical model, which runs through all aspects of the perioperative period. Its successful implementation requires the collaboration of a multidisciplinary team of surgeons, anesthesiologists, nurses, dietitians and rehabilitation therapists, as well as the collaboration of patients and their families. Acute Stanford type A aortic dissection is difficult and has A high incidence of postoperative complications. The improvement of surgical techniques alone is not enough to improve the therapeutic effect of acute Stanford Type A aortic dissection. Although with the progress of surgical techniques, intraoperative extracorporeal circulation and organ protection techniques, the incidence of early postoperative complications after acute Stanford Type A aortic dissection has decreased, it is still higher than other cardiac operations, and the occurrence of early postoperative complications can significantly increase the postoperative mortality [23]. Therefore, to improve the therapeutic effect of acute Stanford type A aortic dissection, in addition to improving surgical techniques, it is necessary to improve the understanding and prevention level of early postoperative complications. Clarifying the risk factors of early postoperative complications is not only helpful to take measures to avoid early postoperative complications, but also helpful to predict the occurrence probability and guide targeted treatment. 
ERAS' application in the surgery of acute Stanford type A aortic dissection still needs to deepen the understanding of early postoperative complications, so as to further improve the ability to prevent and treat complications. However, the published papers on the application of ERAS in the surgery of acute Stanford Type A aortic dissection still have limited evidence, and ERAS schemes in different studies differ greatly, so no unified scheme and standards have been formed yet. Only one study has elaborated on the compliance of ERAS schemes. Meanwhile, previous clinical studies on ERAS were mostly led by surgeons, focusing on the improvement of surgical methods, while studies led by nurses were rarely reported, which led to the ineffective implementation of relevant measures of ERAS, thus affecting the implementation effect and the outcome of patients.

\section{Current Situation of Clinical Application of Cluster Nursing}

Bundles of Care are the integration of evidence-based treatments and nursing interventions for the treatment of complex clinical diseases and the improvement of the quality of medical care, thereby improving patient outcomes [24]. Cluster nursing usually includes 3 - 6 simple and clear evidence-based practices, each of which has been proven to improve patient outcomes through clinical practice and has strong operability. Cluster nursing is an effective approach that closely combines evidence-based research with clinical practice, and its joint implementation has a better clinical intervention effect than the individual implementation of each measure. The implementation of cluster regimens is neither mandatory nor fixed. Different cluster regimens should be adopted according to patients' disease types and conditions. Meanwhile, they will be further improved with the deepening of clinical practice and the generation of new evidence-based evidence. The concept of cluster intervention was first proposed by the United States Institute of Health Care Quality Improvement in 2001. It was first applied to prevent ventilators associated pneumonia and introduced into the field of nursing, and its effectiveness has also been verified [25]. In recent years, with cluster of nursing concept promotion and experts at home and abroad to continuously explore, cluster has wide application in the first aid, the nursing for severe acute pancreatitis, malignant tumor, pressure sores, sepsis and venipuncture clinical nursing fields and achieved good clinical effect [26] [27], in promoting cluster of nursing development in clinical nursing practice at the same time, also shortened the $\mathrm{q}$ of the scientific research and clinical practice. In recent years, with the development of evidence-based nursing in China, the application of cluster nursing in the field of foreign nursing has been relatively mature, and with the development of evidence-based nursing practice in China, cluster nursing has been more and more applied in clinical practice, to promote the continuous improvement of nursing quality and patient outcomes. The introduction of cluster nursing in China is relatively late, and the related research 
is still in the exploratory stage. It is mainly applied in the prevention and control of infection, and further research is needed in the perioperative management and other fields. At the same time, there are also some problems in the clinical practice of cluster nursing [28]. For example, evidence-based nursing is ignored in the construction process of cluster nursing scheme; The measures are not sufficiently interconnected; For every measure of cluster care solutions is immutable, ignored the will go along with the development of clinical practice and improve cluster nursing plan to build a process description is not clear, etc. [29], one of the most important problem is that nursing is the simple clinical nursing measures of cluster of overall and lack of evidence-based basis, should be improved in future research.

\section{Clinical Strategies of ERAS Nursing in Hepatectomy}

\subsection{Identify Evidence-Based Issues}

PICO format was used to construct the evidence-based problems, where $\mathrm{P}$ was the specific population, I was the intervention, $\mathrm{C}$ was the control/Comparator and $\mathrm{O}$ was the outcome. Based on the literature review and clinical practice, the evidence-based question was determined as follows: does cluster care in accelerated rehabilitation surgery promote postoperative recovery in PATIENTS with TAAD compared with traditional care?

\subsection{Develop ERAS Cluster Nursing Strategies for TAAD Patients}

3 - 6 specific and operational ERAS nursing intervention measures were selected based on evidence-based research, expert consultation and field investigation to form ERAS cluster nursing strategies and submit them to experts for review and unanimous approval of experts. Finally, ERAS cluster nursing strategies for aortic dissection surgery were determined.

\subsection{Construction and Management of ERAS Cluster Nursing Team}

According to the principle of voluntary selection of department backbone nurses as team members, the formation of accelerated rehabilitation surgery cluster nursing team, and the support and cooperation of relevant medical staff, researchers participate in the whole process. Invite experts from relevant disciplines to discuss and deal with problems in clinical practice that cannot be solved by nurses or procedures that need to be optimized and improved.

\subsection{Training and Assessment of ERAS Cluster Nursing Team}

The team leader will organize thematic study and regular training, and conduct program training and assessment for team members, so as to ensure that team members can master ERAS cluster nursing strategy of aortic dissection. Meanwhile, the head nurse will organize centralized learning, train and assess general nurses with ERAS cluster nursing strategies, and obtain the recognition and support of other nursing staff, so as to ensure the homogeneity of nursing measures. 


\subsection{Clinical Intervention}

ERAS cluster nursing strategy of TAAD surgery was implemented by the patients, including: preoperative health education; Shortening preoperative fasting time and preoperative oral carbohydrate; Mechanical bowel preparation was not performed routinely. Heat preservation and prevention of deep vein thrombosis during operation; Early postoperative oral feeding: early postoperative ambulation, etc.

\subsubsection{Preoperative Care}

Preoperative education: According to statistics, about $80 \%$ of surgical patients will have anxiety before surgery, and about $68 \%$ of patients will have depression. Aortic dissection itself dangerous illness, surgery is difficult, slow recovery, much complications, preoperative education in addition to the conventional process need to patients with surgery and anesthesia, to dispel the fear of patients for surgery and anesthesia and surgery in the intensive care unit (ICU) the matters needing attention and the significance of early postoperative activities, eating and how to cooperate, to increase the confidence of the patients and the plan implementation of compliance. Intestinal preparation: ERAS cluster group fasted $6 \mathrm{~h}$ before surgery, banned water at $2 \mathrm{~h}$, and took $250 \mathrm{ml}$ carbohydrates orally at $2 \mathrm{~h}$ before surgery. Drug management: Intramuscular injection of diazepam was given at 10:00 p.m. one night before surgery to ensure patients' sleep, and subcutaneous intramuscular injection of morphine (5 - $10 \mathrm{mg}$ ) was administered 30 min before surgery to reduce anxiety, so as to prevent excessive tension from causing blood pressure rise and rupture of the interlayer.

\subsubsection{Intraoperative Management}

During the operation, blood recovery should be strengthened. During the operation, blood should be sucked into the blood storage tank by autologous blood recovery pipeline to reduce blood loss. Strengthen the temperature management, pay attention to keep warm during the operation, adopt comprehensive temperature protection technology, adjust the room temperature at $23^{\circ} \mathrm{C}$ and humidity at $55 \%-62 \%$, and lower the temperature to $30^{\circ} \mathrm{C}$ during extracorporeal circulation. The time-varying temperature blanket was adjusted to $38^{\circ} \mathrm{C}$ for reheating, and the temperature of the patient was raised to above $35^{\circ} \mathrm{C}$. The reheating process should be gentle, and the temperature of the limbs should be closely observed, and the peripheral circulation should be noted. After anesthesia, patients were placed with radial artery catheterization, central venous catheterization (CVC), and catheters to reduce irritation.

\subsubsection{Postoperative Care}

1) Multi-mode sedation and analgesia: postoperative pain is one of the important factors affecting the early postoperative activity of patients, and then the postoperative recovery. In 2015, expert Consensus on perioperative pain Management of General Surgery emphasized that postoperative pain was an impor- 
tant stress factor, and pain had adverse effects on postoperative rehabilitation of patients. It emphasized that multi-mode pain relief should be avoided or opioid analgesics should be avoided as far as possible. Surgical trauma, aortic dissection with breathing machine time is relatively long, I adopt comprehensive measures, such as patients with breathing machine during continuous fully calm, avoid intubation to the discomfort of the patients, continuous pumping pain medications, with patients felt no wound pain advisable, immediately after surgery for breast with fixed wound, to reduce the turning or cough bring pain, promote wound healing. After tracheal intubation is removed, a controlled analgesia pump can be used to relieve pain as needed. Whereas traditional analgesia is to use analgesics after patients feel pain, ERAS concept has the advantage of early-oriented treatment in perioperative pain management of patients. 2) postoperative respiratory management: application based on the physiological parameters of tube drawing program, with the patient's own physiological parameter to decide whether to pull out endotracheal intubation, including the patient's body temperature, mental state and lung function, the patient awake after such as stable vital signs as early as possible to take off the breathing machine and assist patients take back, row of phlegm, expectoration drainage does not reach the designated position, prescribed atomization inhalation therapy, if sustained atomizing viscous sputum use the warm boiling water, dilute sputum, easy to produce, when necessary row row of phlegm, phlegm machine or sputum suction in accordance with the doctor's advice. Instruct client to blow up balloon to facilitate lung expansion. 3) Early feeding: after aortic dissection, traditional feeding is nasogastric feeding during endotracheal intubation. A small amount of water is consumed 6 hours after endotracheal intubation is removed, and nutritional supply is mainly satisfied by nasofeeding. ERAS cluster group patients began to drink small amounts of water 2 hours after tracheal intubation was removed, and patients could enter through the mouth without coughing. Early eating after tracheal intubation was removed could stimulate the gastrointestinal tract and promote the recovery of intestinal peristalsis. Early extubation 4): postoperative patients with aortic dissection compared with other surgical will carry more lines, pericardium, mediastinum and chest drainage tube, dynamic and venous catheter, nasogastric tube, catheter on more lien increased the risk of infection, strengthen the discomfort, limit postoperative activity, and the recovery of gastrointestinal function in patients with delayed, increase the risk of complications, so on the basis of the guarantee adequate drainage, surgical effect should create opportunities, as far as possible pull out as soon as possible, in order to promote recovery. 5) Early activities Studies have shown that even absolute bed rest in healthy people can lead to decreased muscle strength, decreased muscle strength by $1 \%$ to $3 \%$ every day, decreased muscle strength by $10 \%$ to $15 \%$ every week, and decreased to $50 \%$ of the original muscle strength after 3 to 5 weeks. Long-term postoperative bed will increase the risk of lower limb venous thrombosis, pulmonary infection, and is not conducive to gastroin- 
testinal peristalsis. Patients in ERAS group of our department assisted to move their lower limbs in bed every day during postoperative tracheal intubation, and passive exercise was performed once every $4 \mathrm{~h}$, with each time lasting for $30 \mathrm{~min}$. Turn the body and pat the back once every $2 \mathrm{~h}$; Sufficient analgesia was given after tracheal intubation was removed. On that day, the lower limbs were lowered to the bedside and began to step on a bench for exercise, twice a day for 30 min each time. On the second day, 2 nurses assisted bedside activities, 3 times a day for 20 minutes each time. Patients were encouraged to do ankle pump exercise on the bed by themselves. After TAAD, there are many kinds of pipelines for patients, so each pipeline should be properly fixed before each activity.

\subsubsection{Discharge Guidance}

General hospital patients by the nurse in the hospital before do conventional discharge guidance, including detailed considerations, the guidelines, the review time, etc., ERAS cluster is changed when the group of patients discharged from hospital in addition to the regular guidance through the establishment of "protection with" one to one WeChat continuity care guide relationship, solving all patients discharged from hospital after a series of problems, timely remind patients and review of the continuity of care services guidance of the competent deputy director of the doctor, the doctor, the head nurse, nurse, at any time to solve any needs of the patients after discharge.

\section{Summary}

To sum up, ERAS and cluster nursing are both based on new ideas based on evidence. Their purpose is to improve the quality of medical treatment and care, thus improving the clinical outcome of patients. This study adopts the method of evidence-based nursing practice to formulate conforms to our country actual clinical acute Stanford type A aortic dissection (TAAD), the ERAS of cluster nursing strategy, promote the application of the evidence-based nursing in clinical practice, give full play to the leading role of the nursing, the nursing staff to participate in the patients with clinical decision with the doctor. Therefore, further research should be carried out on the clinical role of nurses in ERAS program, and ERAS care program that conforms to China's national conditions should be formulated so that each link of ERAS program can be closely linked, thus ERAS can promote patient surgery.

Thus, ERAS can promote postoperative recovery of patients, reduce postoperative complications and shorten hospital stay. At the same time, it provides reference basis for the improvement of perioperative nursing measures for TAAD patients and scientific basis for future research.

\section{Foundation Project}

Hainan Health Commission Science and Technology Project (No. 20A200004) and Supported by Hainan Natural Science Foundation (Project No. 817393). 


\section{Conflicts of Interest}

The authors declare no conflicts of interest regarding the publication of this paper.

\section{References}

[1] Zhang, J.Z. and Li, X. (2017) Perioperative Accelerated Rehabilitation and Surgical Nursing Care for Patients with Heart Valve Replacement. Journal of Nursing, 32, 27-30.

[2] Sun, L.Z., Liu, N.N., Chang, Q., et al. (2003) Refinement Classification of Aortic Dissection and Its Application. Chinese Journal of Surgery, 43, 7-12.

[3] Sun, L.Z., Qi, R.D., Zhu, J.M., et al. New Standards for Treatment of Complex Type A Aortic Dissection. Chinese Medicine, No. 6, 1-3.

[4] Sun, L.Z. (2012) Aortic Surgery. People’s Medical Publishing House, Beijing, 127.

[5] Gao, H. (2014) Nursing Progress in the Application of Accelerated Rehabilitation Surgery in Cardiac Surgery. Tianjin Nursing, 22, 92-93.

[6] Xiao, W. (2012) Application and Prospect of Rapid Rehabilitation Medicine in Cardiac Surgery. Journal of Applied Medicine, 28, 10-12.

[7] Zhuang, C.L., Ye, X.Z., Zhang, X.D., et al. (2013) Enhanced Recovery after Surgery Programs versus Traditional Care for Colorectal Surgery: A Meta-Analysis of Randomized Controlled Trials. Diseases of the Colon \& Rectum, 56, 667-678. https://doi.org/10.1097/DCR.0b013e3182812842

[8] Ansari, D., Gianotti, L., Schroder, J., et al. (2013) Fast-Track Surgery: Procedure Specific Aspects and Future Direction. Langenbeck's Archives of Surgery, 398, 29-37. https://doi.org/10.1007/s00423-012-1006-9

[9] Cerantola, Y., Valerio, M., Persson, B., et al. (2013) Guidelines for Perioperative Care after Radical Cystectomy for Bladder Cancer: Enhanced Recovery after Surgery (ERAS ((R))) Society Recommendations. Clinical Nutrition, 32, 879-887. https://doi.org/10.1016/j.clnu.2013.09.014

[10] Dasari, B.V., Rahman, R., Khan, S., et al. (2015) Safety and Feasibility of an Enhanced Recovery Pathway after a Liver Resection: Prospective Cohort Study. HPB (Oxford), 17, 700-706. https://doi.org/10.1111/hpb.12447

[11] Wick, E.C., Shore, A.D., Hirose, K., et al. (2011) Readmission Rates and Cost Following Colorectal Surgery. Diseases of the Colon \& Rectum, 54, 1475-1479. https://doi.org/10.1097/DCR.0b013e31822ff8f0

[12] Nygren, J., Thacker, J., Carli, F., et al. (2013) Guidelines for Perioperative Care in Elective Rectal/Pelvic Surgery: Enhanced Recovery after Surgery (ERAS ((R))) Society Recommendations. World Journal of Surgery, 37, 285-305. https://doi.org/10.1007/s00268-012-1787-6

[13] Kong, Z.W., Li, J.S., Wang, Z.M., et al. (2007) Study on the Safety and Efficacy of Accelerated Surgical Therapy for Gastric Cancer. Chinese Journal of Surgery, No. 19, 1314-1317.

[14] Lemanu, D.P., Singh, P.P., Berridge, K., et al. (2013) Randomized Clinical Trial of Enhanced Recovery versus Standard Care after Laparoscopic Sleeve Gastrectomy. British Journal of Surgery, 100, 482-489. https://doi.org/10.1002/bjs.9026

[15] Lv, L., Shao, Y.F. and Zhou, Y.B. (2012) The Enhanced Recovery after Surgery (ERAS) Pathway for Patients Undergoing Colorectal Surgery: An Update of Meta-Analysis of Randomized Controlled Trials. International Journal of Colorectal 
Disease, 27, 1549-1554. https://doi.org/10.1007/s00384-012-1577-5

[16] Hughes, M.J., Mcnally, S. and Wigmore, S.J. (2014) Enhanced Recovery Following Liver Surgery: A Systematic Review and Meta-Analysis. HPB (Oxford), 16, 699-706. https://doi.org/10.1111/hpb.12245

[17] Bona, S., Molteni, M., Rosati, R., et al. (2014) Introducing an Enhanced Recovery after Surgery Program in Colorectal Surgery: A Single Center Experience. World Journal of Gastroenterology, 20, 17578-17587. https://doi.org/10.3748/wig.v20.i46.17578

[18] Varadhan, K.K., Neal, K.R., Dejong, C., et al. (2010) The Enhanced Recovery after Surgery (ERAS) Pathway for Patients Undergoing Major Elective Open Colorectal Surgery: A Meta-Analysis of Randomized Controlled Trials. Clinical Nutrition (Edinburgh, Scotland), 29, 434. https://doi.org/10.1016/j.clnu.2010.01.004

[19] Mortensen, K., Nilsson, M., Slim, K., et al. (2014) Consensus Guidelines for Enhanced Recovery after Gastrectomy: Enhanced Recovery after Surgery (ERAS(R)) Society Recommendations. British Journal of Surgery, 101, 1209-1229. https://doi.org/10.1002/bjs.9582

[20] (2015) Aftershocks. Application of Accelerated Rehabilitation Surgery in Gastric Cancer Surgical Towels. Chinese Journal of Digestive Surgery, 14, 33-37.

[21] Ren, N.N., Zhang, Y.M., Liu, A., et al. (2015) Meta-Analysis on the Effect of Accelerated Rehabilitation Surgical Nursing on the Rehabilitation Effect of Patients Undergoing Subtotal Gastrectomy. Journal of Nursing, No. 16, 98-102.

[22] Xiao, B.Y., Wang, Y.Q., Cao, H.H., et al. (2016) Clinical Analysis of Perioperative Application of the Concept of "Accelerated Rehabilitation Surgery" in Elderly Patients with Gastric Cancer. Chinese Journal of Clinicians (Electronic Edition), 1-2.

[23] Shang, W., Liu, N., Yan, X.L., et al. (2011) Analysis of Early Complications after Type A Aortic Dissection. Journal of Cardiopulmonary Diseases, 3, 183-186.

[24] Wang, Z., Jiang, M. and Yang, L.P. (2012) Study on the Application of Cluster Nursing of Digestive Tract Poison Removal in the Treatment of Acute Severe Organophosphorus Poisoning. Journal of Nursing Advancement, No. 5, 389-391.

[25] Chen, M.H., Chen, Q.L., Song, D.D., et al. (2014) Application of Cluster Nursing in Reducing the Incidence of Pressure Ulcers in Patients with Unavoidable Pressure Ulcers. Journal of Nursing, No. 10, 43-44.

[26] Zhang, J. and Ning, X.D. (2014) Application of Cluster Nursing Strategy in Patients with Central Venous Catheterization. Journal of Nursing, No. 2, 17-20.

[27] Rhodes, D., Cheng, A.C., Mclellan, S., et al. (2016) Reducing Staphylococcus aureus Bloodstream Infections Associated with Peripheral Intravenous Cannulae: Successful Implementation of a Care Bundle at a Large Australian Health Service. The Journal of Hospital Infection, 94, 86-91. https://doi.org/10.1016/j.jhin.2016.05.020

[28] Wu, M.B. and Hu, Y. (2013) The Error Analysis and Correct Application of Cluster Nursing. Journal of Nursing, No. 18, 84-86.

[29] Crunden, A., Boyce, C., Woodman, A., et al. (2005) An Evaluation of the Impact of the Ventilator Care Bundle. Nursing in Critical Care, 10, 241-246.

https://doi.org/10.1111/j.1362-1017.2005.00134.x 